\title{
Tool use by foraging ants
}

\section{H. Richards ${ }^{1}$}

Published online: 28 February 2022

(c) International Union for the Study of Social Insects (IUSSI) 2022
In 1960, Jane Goodall watched a chimpanzee in Gombe turn a twig into a fishing tool for termites. With that observation, tool use was relegated to the ever-growing list of traits that do not make us human. Since then, the list of animals that use tools has been growing, and definitions of what constitutes tool use have also changed. Current definitions generally describe tools as objects derived from the environment that animals use to modify or adjust other objects or themselves (Bentley-Condit 2010). The best known animal tool users are vertebrates, of course. Perhaps that is why the phylogenetic breadth of animal tool use remains under-appreciated, and why it is still surprising to learn that tool use also occurs in at least 38 genera of invertebrates, including molluscs, crustaceans, and insects, (Bentley-Condit 2010). Among insect tool users are eight ant genera, in which tool use is mainly associated with foraging behaviour.

Ant tool use was first documented in four Aphaenogaster species that use tools to carry liquid food to their nests (Fellers and Fellers 1976). These ants lack extensible crops, so are unable to carry large volumes of liquids internally. This means that when they discover liquid or very soft food sources, they cannot efficiently carry the food back to their nests. A solution to this problem is to use a tool that, like a sponge, can absorb or be coated with the food and then carried home. Ants accomplish this by dropping grains of soil, sand, pieces of leaf, small twigs, or other absorbent objects into the liquid food, waiting a short time for the tool to absorb the food, then retrieving it and carrying it to the nest. Ants that can use tools like this not only might forage more efficiently, but might also be more competitive against other ants using the same food sources, but without tools (Fellers and Fellers 1976). Subsequent studies of Aphaenogaster have suggested that within colonies, only some workers use tools (Banschbach et al. 2006), that they are selective in the tools that they choose, and that tool usage can improve with

M. H. Richards

mrichards@brocku.ca

1 Brock University, St. Catharines, ON, Canada experience (Maák et al. 2017). In some cases, ant workers even use pieces of sponge for this task, cutting the sponge into apparently more manageable pieces (Maák et al. 2017).

In this issue, Gábor Módra and colleagues continue their studies of foraging tool use by ants, with a comparison of two species with different foraging habits (Módra et al. 2021). They test the hypothesis that tool use should be more frequently observed in ants in which soft or liquid food, such as honeydew, rotten fruit or liquefying corpses of arthropods, represents a significant portion of their diet. Granivores, such as Messor structor that rarely exploit liquid foods would have little need for tools, and so might be expected to use them less frequently or not at all. In contrast, omnivores such as Aphaenogaster subterranea that do exploit liquid food sources could benefit substantially from using tools, and so would be expected to use tools more frequently. Módra and colleagues predicted that Aphaenogaster workers would use tools sooner and more frequently than Messor workers, and would also be choosier about which tools they selected.

Módra et al.'s study was carried out in a laboratory setting. Five colonies of each species were collected and established in plastic boxes connected by tunnels to a foraging area where foraging workers could be observed. After being starved for four days to ensure that they would be hungry, ants were supplied with honey-water and six types of objects that could be used as tools: sand grains, small and large soil grains, pieces of pine needles, pieces of leaves, and pieces of sponges. The tools were mixed together and placed near the honey-water baits, as previous studies suggest that ants are more likely to use tools near the food source. Once the first workers found the honey-water, observations commenced, continuing for $5 \mathrm{~h}$. As predicted, Aphaenogaster colonies dropped and retrieved tools much more often than Messor colonies. Their latency to tool usage was also much shorter-they dropped and retrieved tools sooner in the experiments. In fact, Aphaenogaster colonies used tools so efficiently that they used up all their food by the end of the 5-h experiment, whereas Messor colonies consumed only a small portion of their food. In other words, having 
appropriate tools for carrying home the groceries, really did enhance foraging efficiency.

An enduring mystery is how this tool use behaviour came to evolve. One possibility is that before objects such as sand grains were used as sponges, they were used to cover or hide food sources from competitors. Another proposal is that ants could prevent entanglement of foragers in sticky messes by covering them with small objects. Addressing these hypotheses would require observations of tool use in natural settings, but as yet, there is almost no direct evidence of ant tool use in natural settings without artificial baits. Observations of ants licking or carrying objects with absorbed food are not enough to demonstrate tool use-it is necessary to demonstrate that the ants select objects, put them in the food, and then carry the objects back to their nests. Field studies of ant tool use would undoubtedly be challenging, but perhaps a first step would be inducing field colonies to forage at food baits near nest entrances.

\section{References}

Banschbach VS, Brunelle A, Bartlett KM et al (2006) Tool use by the forest ant aphaenogaster rudis: ecology and task allocation. Insect Soc 53:463-471. https://doi.org/10.1007/s00040-006-0897-2

Bentley-Condit V (2010) Animal tool use: current definitions and an updated comprehensive catalog. Behaviour 147:185-232. https:// doi.org/10.1163/000579509X12512865686555

Fellers JH, Fellers GM (1976) Tool use in a social insect and its implications for competitive interactions. Science 192:70-72. https:// doi.org/10.1126/science.192.4234.70

Maák I, Lôrinczi G, Le Quinquis P et al (2017) Tool selection during foraging in two species of funnel ants. Anim Behav 123:207-216. https://doi.org/10.1016/j.anbehav.2016.11.005

Módra G, Maák I, Lőrincz Á, Lőrinczi G (2021) Comparison of foraging tool use in two species of myrmicine ants (Hymenoptera: Formicidae). Insect Soc. https://doi.org/10.1007/s00040-021-00838-0 